\title{
Propuesta de actividades de participación activa para el acercamiento de la Ingeniería Civil a estudiantes de secundaria
}

\author{
Aitor Fernández-Jiménez ${ }^{\mathrm{a} 1}$, Eduardo Álvarez-Álvarez ${ }^{\mathrm{a} 2}$, Rodolfo Espina-Valdés ${ }^{\mathrm{a} 3}$, Antonio \\ Navarro-Manso ${ }^{\text {a4 }}$, Víctor Manuel Fernández-Pacheco ${ }^{\text {a5 }}$ \\ aÁrea de Ingeniería Hidráulica, Escuela Politécnica de Mieres, Universidad de Oviedo, Calle Gonzalo Gutiérrez \\ Quirós s/n, 33600 Mieres, fernandezaitor@uniovi.es ${ }^{1}$, edualvarez@uniovi.es $^{2}, \underline{\text { espinarodolfo@uniovi.es }}^{3}$, \\ navarroantonio@uniovi.es ${ }^{4}$, fernandezpvictor.uo@uniovi.es $^{5}$
}

\begin{abstract}
The realization of activities applying an active participation methodology allows a better assimilation of concepts and promotes autonomous work by the students. These methodologies require more effort by the teaching team, but new skills are retained much more time. This article presents a practical activity to approach civil engineering and university environment to secondary education students, who are close to start their higher education stage. The activity was carried out on an engineering olympiad format and consisted on two different tests: one related to hydraulics and the other one related to structures and bridges. At the end of the activity students filled a satisfaction survey, obtaining very possitive results. The collected suggestions will serve to improve this activity in future editions.
\end{abstract}

Keywords: Civil engineering, active participation, engineering olympiad, loose materials' dam, bridge model

\section{Resumen}

La realización de actividades aplicando una metodología basada en la participación activa permite una mejor asimilación de conceptos y promueve el trabajo autónomo por parte de los estudiantes. Estas metodologías requieren de más esfuerzo por parte del equipo docente, pero las nuevas habilidades se retienen por mucho más tiempo. Este artículo presenta una actividad práctica para acercar la Ingeniería Civil y el ambiente universitario a los estudiantes de educación secundaria que se encuentra próximos a iniciar su etapa en la educación superior. La actividad se llevó a cabo en un formato de olimpiada de ingeniería y consistió en la realización de dos pruebas diferentes: una relacionada con la hidráulica y otra relacionada con las estructuras y puentes. Al finalizar la actividad, los estudiantes cumplimentaron una encuesta de satisfacción cuyos resultados han sido muy positivos. Estas sugerencias servirán para mejorar algunos aspectos de la prueba en futuras ediciones.

Palabras clave: Ingeniería Civil, Participación activa, olimpiada ingeniería, presa de materiales sueltos, maqueta de puente 


\section{Introducción}

La Ingeniería Civil es la especialidad de la ingeniería que se dedica exclusivamente al diseño, construcción y mantenimiento de diversas infrarestructuras como carreteras, canales, embalses, puentes, ferrocarriles o puertos (CITOPIC). Normalmente, estas infraestructuras son utilizadas por la mayor parte de la sociedad de una región o país, por lo que tienen una gran importancia económica y social. Sin embargo, la multidisciplinariedad de esta rama de la ingeniería hace que sea bastante desconocida para el conjunto de la población y, más en concreto, por parte de los alumnos que aún no han accedido a la universidad. Por ello, es básico realizar actividades que pretendan dar publicidad y acercar al nuevo público los ámbitos donde se aplica la Ingeniería Civil.

La realización de actividades prácticas donde exista un aprendizaje activo por parte de los alumnos ayuda a interiorizar y clarificar ideas, lo que resulta muy útil para concretizar conceptos. El aprendizaje activo se relaciona con metodologías que invitan a la proactividad por parte del estudiante, de modo que éste razone autónomamente durante el desarrollo de la actividad práctica planteada (Prince, 2004). Estas metodologías requieren de un mayor esfuerzo por parte de los estudiantes y de los profesores, pero suelen obtenerse muy buenos resultados y valoraciones en comparación con otras metodologías tradicionales (León et al, 2011). Tanto es así, que algunas experiencias llevadas a cabo aseguran que puede favorecer a que los conocimientos adquiridos se mantengan mucho más en el tiempo (Lacuesta et al, 2009).

Este tipo de actividades han sido realizadas en otros centros educativos con el objetivo de acercar las enseñanzas superiores a alumnos de secundaria o de formación profesional que se encuentran interesados en cursar una ingeniería. Destacan algunas experiencias como la preparación de una Olimpiada Matemática por parte del grupo de investigación de Matemáticas Aplicadas a la Ingeniería Civil (MAIC) de la Universidad Politécnica de Madrid que desarrolló en 2010 un evento para alumnos de instituto con muy buena acogida (MAIC, 2010). También destaca la I Olimpiada de Ingeniería Civil de la Escuela Politécnica de Cartagena (UPCT) que a principios de 2019 organizó un evento para acercar la ingeniería civil a los alumnos que se encontraban en condiciones de presentarse a la Evaluación del Bachillerato para el Acceso a la Universidad (EBAU). En ella, se planteaba un problema relacionado con la ingeniería civil que deberían de resolver los alumnos de forma autónoma (UPCT, 2019). Por último, la Universidad de Castilla la Mancha realizó en 2010 una Olimpiada de Informática donde mediante la elaboración de una gymkana se pretendía dar visibilidad de los estudios de ingeniería informática a alumnos preuniversitarios (Alfaro et al, 2011).

Relacionadas directamente con la ingeniería civil destacan las Olimpiadas del Colegio de Ingenieros de Caminos que se vienen celebrando desde el año 2018. En ella participan alumnos de diferentes provincias de $1^{\circ}$ y $2^{\circ}$ de Educación Secundaria Obligatoria (ESO), desarrollándose en dos fases: una primera local entre institutos de la región adscrita y una final a nivel nacional entre los equipos ganadores (UPCT, 2020). En ellas, se ponen en práctica diferentes temáticas relacionadas con la ingeniería civil como construcción de un arco de dovelas o un desafío de logística y transporte. A nivel internacional existen algunas experiencias similares en la Universidad de Chile (Asociación Chilena de Estudiantes de Ingeniería-ACHEII, 2016) o la Olimpiada Nacional de Estudiantes de Ingeniería Civil de México (UMSNH, 2018). Estas experiencias fueron desarrolladas por estudiantes de nivel universitario con el objetivo de profundizar en el trabajo en equipo y la competitividad dentro del mundo empresarial.

El presente artículo presenta la actividad práctica planteada por parte del Área de Ingeniería Hidráulica para la II Olimpiada de Ingeniería de la Escuela Politécnica de Mieres (Universidad de Oviedo). Esta actividad fue desarrollada por alumnos de secundaria de centros de enseñanza del Principado de Asturias en una jornada de puertas abiertas de la Escuela. La actividad consistía, por un lado, en la construcción de una presa de materiales sueltos mediante la utilización de una serie de materiales granulares, disponiendo

(cc) BY-NC-ND 2020, Universitat Politècnica de València 
los alumnos de quince minutos en total para su ejecución. Tras la realización de la prueba, el equipo que mejor haya ejecutado el talud y que menos agua haya filtrado resultará ganador del evento. Por otro lado, cada grupo de estudiantes debía traer construida una maqueta de puente para luego ser sometida a una prueba de carga hasta colapso de la estructura. Aquella maqueta que mayor peso resistiese sería la ganadora. Adicionalmente, el jurado de la prueba evaluó otros aspectos estéticos y de ejecución de modo que al mejor equipo se le concedía el premio del jurado.

\section{Objetivos}

La olimpiada en ingeniería civil busca acercar conocimientos científico-técnicos a alumnos de secundaria de un modo lúdico y participativo. Así, se busca que se familiaricen con aspectos clave del ámbito ingenieril, de modo que puedan interesarse en cursarlos en su futuro universitario. Se ha creado un sistema de competición basado en dos pruebas. Por un lado, se pide construir una presa de materiales sueltos y, por otro lado, se busca construir una maqueta de puente utilizando palillos de madera.

Los objetivos principales de esta actividad se basan en la aplicación de metodologías activas de enseñanza de modo que los alumnos aumenten su confianza, mejoraen su capacidad de razonamiento y adquieran criterio técnico a la hora de abordar problemas. Además, puesto que es una actividad grupal se refuerza el sentido de cooperación. Por último, se busca que la actividad resulte divertida y atractiva, valorándose este punto cumplimentando una encuesta de satisfacción por parte de los alumnos al finalizar la actividad.

\section{Desarrollo de la innovación}

\section{Las presas de materiales sueltos}

\subsection{Planteamiento teórico}

Las presas de materiales sueltos son estructuras hidráulicas que se construyen con materiales granulares naturales bien sea escollera, grava, rocas, arenas o arcilla. Esta tipología de presa ha sido utilizada desde la Antigüedad debido a que no requieren de la utilización de ningún tipo de material aglomerante, sino que el simple acopio de diferentes materiales granulares en capas permiten la confección de la presa. Además, estas estructuras suelen ser más baratas que las presas de fábrica ya que son construidas con materiales procedentes de la excavación del vaso.

El mecanismo resistente de cualquier presa de materiales sueltos es la gravedad, siendo el peso propio de los diferentes materiales que la componen los que ofrecen resistencia al empuje del agua. Por otro lado, el volumen y su sección dependerá de la naturaleza de los materiales granulares utilizados, siendo el ángulo de rozamiento interno el factor principal que indica la inclinación máxima admitida por el talud. Cuánto mayor inclinación tenga el paramento, más posibilidades hay de que se produzcan deslizamientos en la cerrada que puedan provocar el colapso de la presa.

Al componerse de una sucesión de capas de materiales granulares, se producen una serie de intersticios entre los diferentes gránulos que componen la estructura. Por lo tanto, la impermeabilidad del cuerpo no está garantizada, requiriéndose del uso de arcillas u otros conglomerados hidráulicos en lugares estratégicos de la estructura que eviten el paso del agua. Según la disposición de los materiales podemos distinguir tres tipos diferentes de presas de materiales sueltos: homogéneas, de pantalla impermeable y de núcleo (ver Fig. 1). 

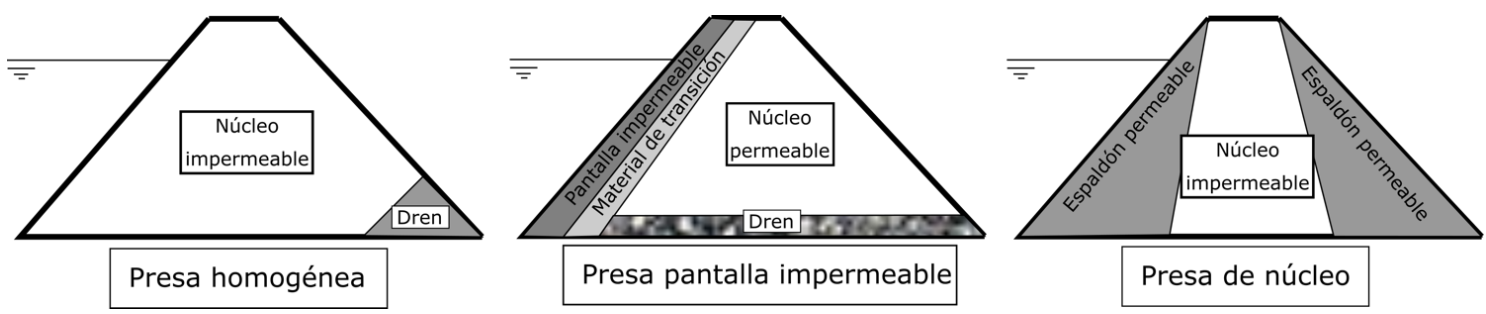

Fig. 1. Tipologías de presas de materiales sueltos. [Elaboración propia].

Los dos criterios críticos a la hora del cálculo y diseño de la estabilidad de las presas de materiales sueltos son la estabilidad del talud y la filtración del agua hacia el interior de la presa. Cabe destacar que para evitar la permeabilidad de la cerrada es imprescindible la selección de materiales aptos para su utilización y que la ejecución de las capas sucesivas se realice de acuerdo a las prescripciones técnicas.

La no correcta selección y control de estas tareas puede avocar en catástrofe, como así ocurrió en 1976 en la presa de 73 metros de altura de Teton (Estados Unidos) (Campos, 2013). El colapso de esta presa de materiales sueltos se produjo durante su primer llenado debido a la utilización de materiales inadecuados (riolita y toba volcánica), lo que produjo una infiltración desmesurada de agua y la destrucción completa de la infraestructura (ver Fig. 2).
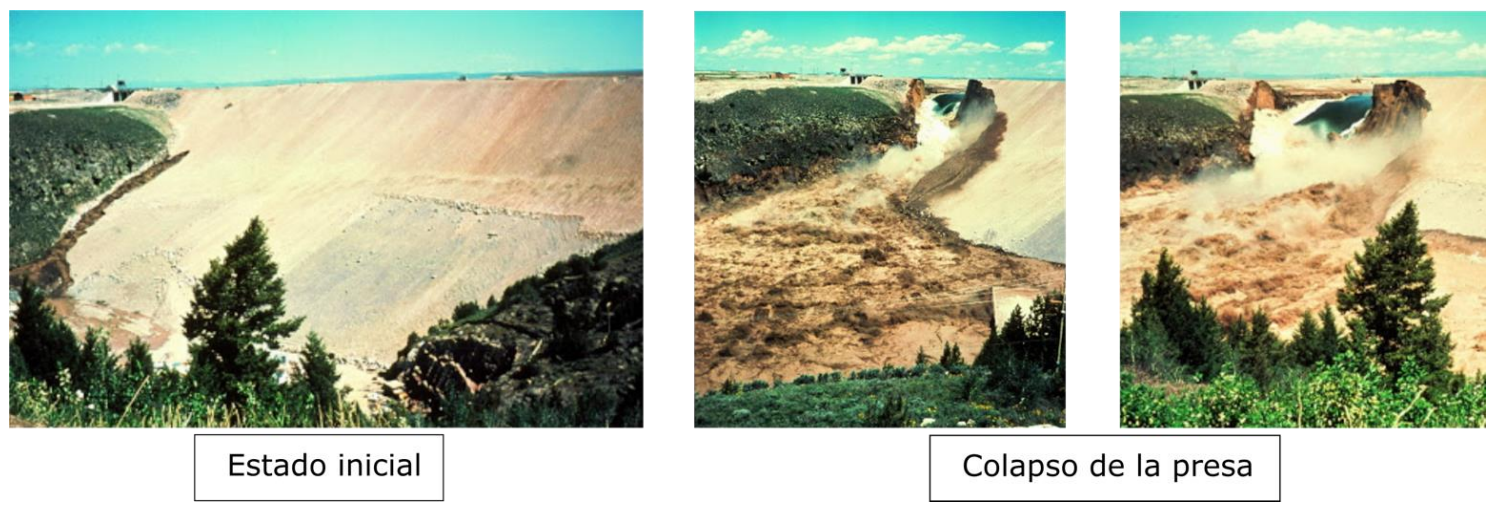

Fig. 2. Cronograma del colapso de la presa de Teton. [Elaboración propia]

Otro criterio adicional a considerar es el diseño de la altura de resguardo de modo que se pueda evitar el vertido y rotura por coronación. La cota de coronación de una presa es la cota más elevada de su estructura resistente mientras que el resguardo es la altura de seguridad que se establece entre la máxima altura de lámina de agua y la cota de coronación. Destaca la rotura en 1982 de la presa de materiales sueltos de Tous (España), cuyo vertido por coronación fue provocado por una gran avenida durante un episodio de tormentas intensas aguas arriba de su cuenca (ver Fig. 3).


Fig. 3. Fotografías de la rotura de la presa de Tous en 1982. [Elaboración propia]. 


\subsection{Planteamiento práctico}

El objetivo de la prueba es la construcción por parte de los alumnos de una presa de materiales sueltos en el interior de un cajón, la cual será posteriormente evaluada ante la filtración de agua a través del cuerpo del talud. Dicho talud debía ser construido por la sucesión de capas formadas por diferentes materiales granulares, aumentando de tamaño de grano a medida que nos alejamos del núcleo.

Para ello, se han utilizado los siguientes materiales: cajón transparente de metacrilato con 30 litros de capacidad, arcilla con alta plasticidad y gravas con granulometrías de $5-15 \mathrm{~mm}$ y $15-30 \mathrm{~mm}$ (Santos et al 2011). Además, se dispondrá de una serie de recipientes con agua potable y otros adicionales para el vertido del material a deshechar tras la realización de las distintas pruebas. En la Fig. 4 se muestra una fotografía con el material utilizado.

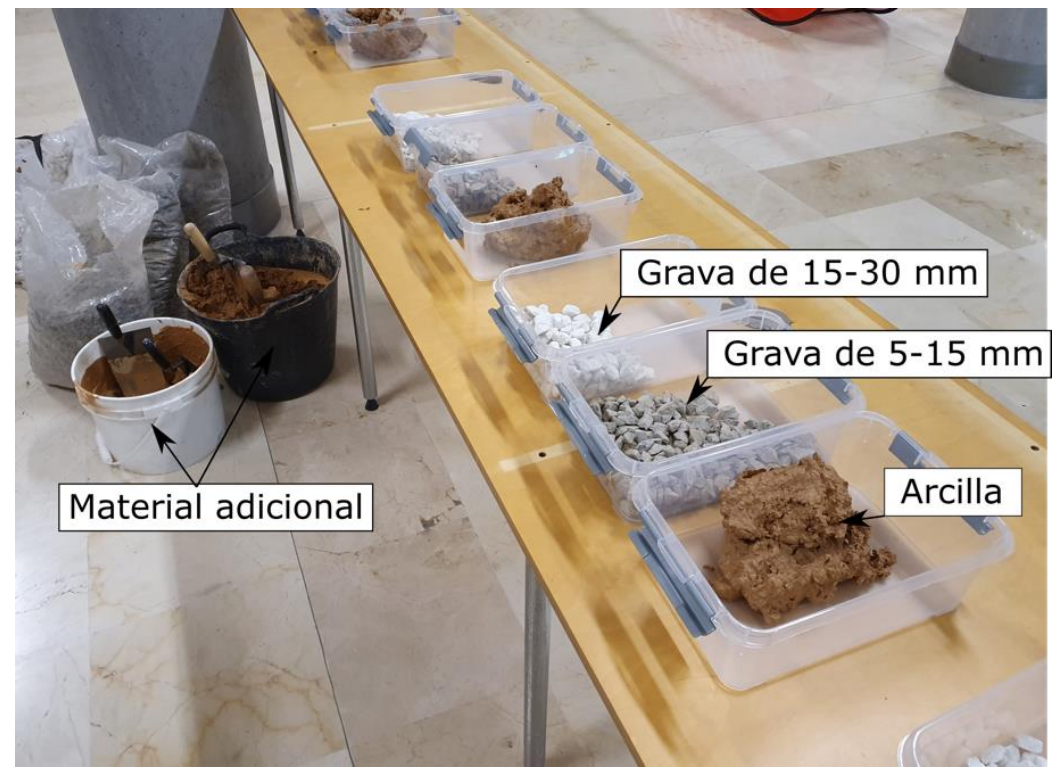

Fig. 4. Fotografia con los materiales utilizados. [Elaboración propia].

La prueba llevada a cabo ha sido cronometrada, disponiendo los alumnos de 5 minutos para la ejecución de la presa de materiales sueltos en el interior del cajón. El volumen de material estaba pre-establecido por el jurado de la prueba, siendo éste previamente tarado de modo que todos los grupos dispusieran de la misma cantidad de material. La altura de coronación del talud es de $15 \mathrm{~cm}$, debiendo ocupar la totalidad del ancho del cajón de plástico.

Una vez se haya finalizado la construcción del talud o, en su defecto, hayan expirado los 5 minutos reglamentarios; se procederá a verter el agua en uno de los paramentos del dique hasta alcanzar una altura de lámina de agua de $10 \mathrm{~cm}$. En ese momento se empieza a cronometrar el tiempo que tarda el agua en filtrarse a través del talud. De este modo, el equipo cuya presa de materiales sueltos más tarde en filtrar el agua habrá ganado la prueba. La Fig. 5 muestra un esquema de la prueba indicando las diferentes fases durante la construcción del talud.

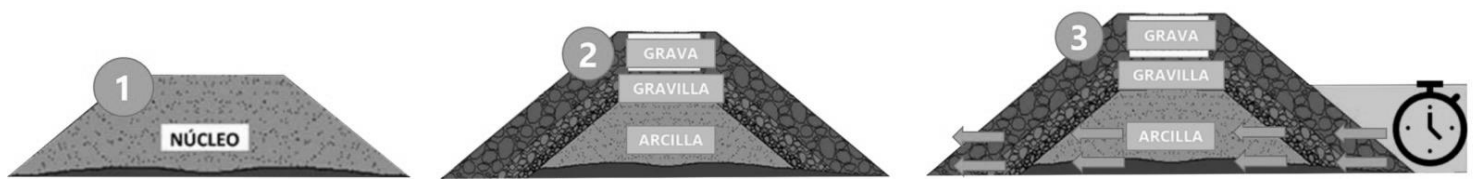

Fig. 5. Fases durante la construcción de las diferentes fases del talud. [Elaboración propia]. 


\section{Los puentes}

\subsection{Planteamiento teórico}

Un puente es una construcción realizada en diferentes materiales como madera, mampostería, acero u hormigón; que se construye para salvar obstáculos como grandes desniveles, cuerpos de agua u otro tipo de infraestructuras (RAE). El proyecto, construcción y mantenimiento de este tipo de infraestructuras es llevado a cabo por ingenieros civiles, aplicándose conocimientos de estructuras, materiales y geotecnia.

Dependiendo de factores como los materiales y técnicas disponibles, la longitud del vano a salvar, la estética o los condicionantes económicos, existen diferentes tipologías. Las principales tipologías de puentes son: de vigas, en arco, en celosía, colgante y atirantados (Yepes, 2017).

Tras la construcción del puente y antes de la puesta en servicio se realiza la denominada prueba de carga. Esta prueba reproduce uno o varios estados de carga para comprobar el diseño y la correcta ejecución del puente. En España, la prueba de carga en puentes de carretera debe realizarse de acuerdo con las Recomendaciones para la realización de pruebas de carga de recepción en puentes de carretera (Ministerio de Fomento, 1999), mientras que para puentes de ferrocarril de más de diez metros de vano será sometido a lo indicado por la Instrucción sobre las Acciones a considerar en el proyecto de Puentes de Ferrocarril IAPF (Ministerio de Fomento, 2007).

Estas pruebas de carga se realizan una vez se ha concluido la ejecución de toda la estructura, por lo que resulta crítica su correcta realización y posterior análisis. En 2015, una pasarela peatonal atirantada de más de 50 m colapsó durante su prueba de carga en Bogotá (Colombia), causando numerosos heridos y costes por más de 1 millón de euros (Estudio Sassani, 2018). Estudios posteriores establecieron que el colapso de la misma vino dado por una sobretensión en los cables de pretensado del tablero de hormigón. La Fig. 6 muestra algunas imágenes del suceso.



Vista transversal

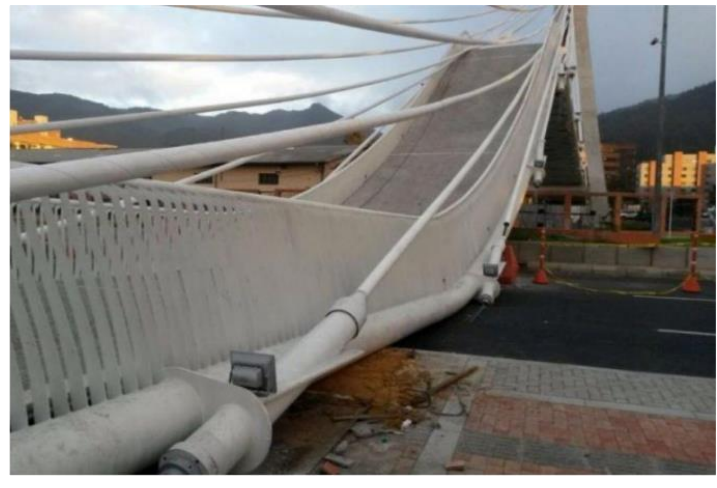

Vista tablero

Fig. 6. Imágenes del colapso de la pasarela peatonal. [Fuente: www.estructurando.net].

\subsection{Planteamiento práctico}

El objetivo principal de esta prueba es la construcción y ensayo de carga hasta la rotura de modelos de puentes construidos con palillos de madera. Para ello, se han proporcionado 750 palillos de madera que tendrán que ser utilizados por los estudiantes para fabricar la maqueta del puente en sus respectivos institutos, utilizando cola blanca en las uniones e hilos de nylon en el caso de que se opte por las tipologías de puente colgante o atirantado. En función de la tipología se utilizará o no una base de madera para asegurar su estabilidad. 
El tablero debía tener una longitud mínima de $70 \mathrm{~cm}$, una anchura comprendida entre 7 y $20 \mathrm{~cm}$ y disponer de un único vano central. El diseño del tablero debía permitir la aplicación de las cargas en toda la superficie. Además, el vano central debía tener una altura mínima de $25 \mathrm{~cm}$. La Fig. 7 muestra una imagen con las dimensiones mínimas requeridas.
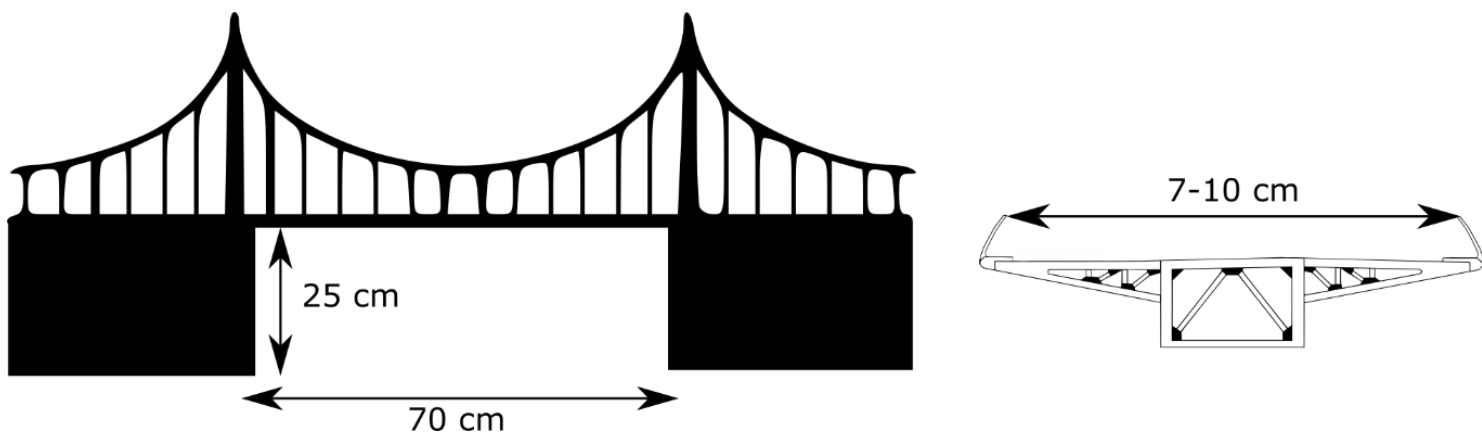

Fig. 7. Dimensiones mínimas de la maqueta a construir. [Elaboración propia].

La prueba de aplicación de carga sobre puente se divide en dos partes bien diferenciadas. En la primera (Parte 1) se aplicaría una carga sobre el tablero del puente hasta el colapso de la estructura, mientras que en la segunda (Parte 2) el jurado evaluaría el diseño, estética y presentación de la maqueta realizada.

La Prueba 1 comienza con la medida y pesaje del puente de modo que se comprueba que la maqueta cumple con los requisitos establecidos, además de permitir estudiar cómo se podría colocar las cargas sobre el tablero. Posteriormente, se procedería a la carga progresiva del puente mediante la utilización de adoquines previamente tarados de 2,5 $\mathrm{kg}$ hasta alcanzar el colapso de la estructura. La carga total resistida vendrá dada por el peso máximo resistido antes de que se produzca el colapso. Para determinar el colapso se deberá haber producido la destrucción total del puente, la rotura de algunos de los elementos portantes principales (tablero, torre, pilar, apoyo o cable) o que la flecha en el centro del vano sea mayor al 3\%.

La Prueba 2 es evaluada por un jurado designado por la organización del evento, valorando los aspectos que se detallan en la Tabla 1 según las puntuaciones establecidas.

Tabla 1. Ponderación de la Prueba 2. [Elaboración propia].

\begin{tabular}{cc}
\hline Aspecto & Puntuación \\
\hline Relación carga/peso & $20 \%$ \\
Estética y funcionalidad & $30 \%$ \\
Acabados & $10 \%$ \\
Presentación de la maqueta & $40 \%$
\end{tabular}

Con todo ello, el equipo cuyo puente mayor peso haya resistido hasta el colapso de la maqueta recibiría el primer premio de la Prueba 1, mientras que aquel que obtenga la mayor puntuación en la Prueba 2 recibiría el premio del Jurado.

\section{Resultados}

La actividad práctica fue desarrrollada por 43 alumnos con edades comprendidas entre los 12 y los 18 años. La creación de los diferentes grupos se realizó según la edad de los participantes para, por un lado, permitir una mejor interacción entre los estudiantes y, por otro lado, para poder segmentar posteriormente sus resultados según edad. Por motivos organizativos, el tiempo máximo permitido para la ejecución de las diferentes pruebas (presa de materiales sueltos y prueba de carga sobre puente) fue de 15 minutos. A 
la finalización de las pruebas por parte de todos los grupos se eligió un equipo ganador. La Fig. 8 muestra el procedimiento constructivo de la presa de materiales sueltos mientras que la Fig. 9 muestra las diferentes maquetas de puente construidas por parte de los alumnos.



Fig. 8. Fotografía de los alumnos construyendo el talud. [Elaboración propia].

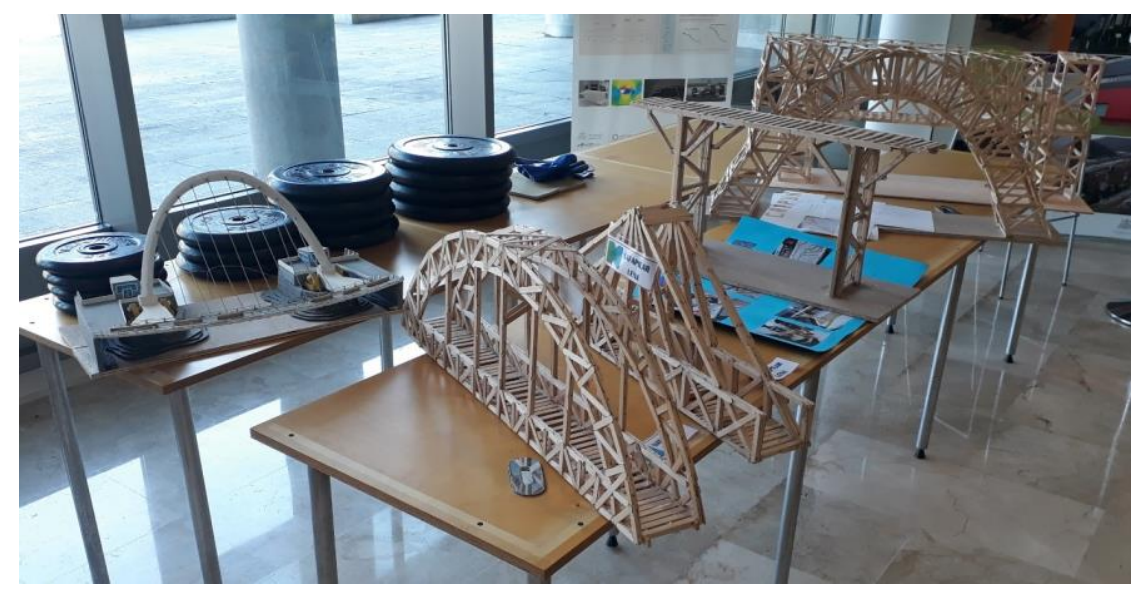

Fig. 9. Exposición de las maquetas de puentes. [Elaboración propia].

Los puentes serían sometidos a la prueba de carga hasta lograr el colapso. En la Fig. 10 se muestran fotografías de los puentes en el banco de ensayos. 

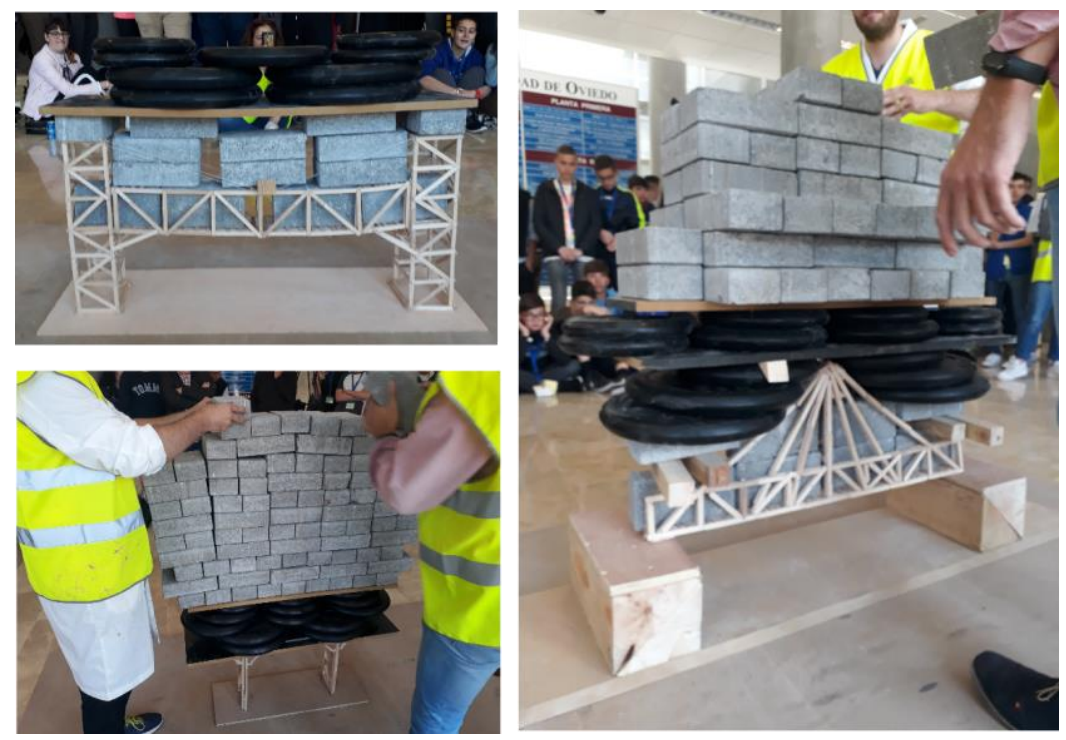

Fig. 10. Fotografias durante la realización de las pruebas de carga. [Elaboración propia].

Tras la realización todas las pruebas, el alumnado participante fue invitado a que cumplimentara una encuesta de opinión. El formulario estaba dividido en tres apartados: desarrollo de la práctica, materialesentorno y otros aspectos. En cada uno de ellos, se realizaban una serie de preguntas, debiendo evaluarse con una puntuación del 1 (menor satisfacción) al 5 (máxima satisfacción). La Fig. 11 muestra la encuesta realizada.

\begin{tabular}{|c|c|c|c|c|c|}
\hline \multicolumn{6}{|c|}{ Desarrollo de h práctica } \\
\hline ASPECTO & 1 & 2 & 3 & 4 & 5 \\
\hline Claridad en la explicación previa & & & & & \\
\hline $\begin{array}{l}\text { Dificultad para identificar el objetivo o de la } \\
\text { actividad planteada }\end{array}$ & & & & & \\
\hline \begin{tabular}{|c}
$\begin{array}{c}\text { Valor ación de los requisitos y normas } \\
\text { de la adtuidad }\end{array}$ \\
\end{tabular} & & & & & \\
\hline $\begin{array}{l}\text { Dificultad a la hora de ufilzar los } \\
\text { diferentes materiales disponibles }\end{array}$ & & & & & \\
\hline \multicolumn{6}{|c|}{ Materiales y entomo } \\
\hline ASPECTO & 1 & 2 & 3 & 4 & 5 \\
\hline $\begin{array}{c}\text { Amabilidad y disponibilidad de la } \\
\text { personas encargadas. }\end{array}$ & & & & & \\
\hline $\begin{array}{l}\text { Accesibilidad y comodidad a la hora de } \\
\text { llev ar a cabo la actividad }\end{array}$ & & & & & \\
\hline \begin{tabular}{|c}
$\begin{array}{c}\text { Grado de satisfación œon el desarrollo } \\
\text { del evento }\end{array}$ \\
\end{tabular} & & & & & \\
\hline \multicolumn{6}{|c|}{ Otros aspectos } \\
\hline ASPECTO & 1 & 2 & 3 & 4 & 5 \\
\hline \multicolumn{6}{|l|}{ ¿Te ha resultado divertida la actividad? } \\
\hline ¿Recomendarías esta acturidad? & & & & & \\
\hline Sugerencias $y$ com entarios: & & & & & \\
\hline
\end{tabular}

Fig. 11. Encuesta de satisfacción. [Elaboración propia].

Los resultados obtenidos para el Bloque 1 (desarrollo de la práctica) indican que tanto el objetivo de las pruebas como la descripción de las normas de participación fueron explicadas a los alumnos de forma clara y concisa, obteniéndose valoraciones de satisfacción máxima superiores al 58\%.

Sin embargo, y aunque tiene una valoración global positiva, la dificultad a la hora de utilizar los diferentes materiales parece que tiene un índice de satisfacción menor. Este aspecto podría justificarse 
debido al reducido conocimiento técnico de estructuras o geotecnia que tienen los alumnos de secundaria, por lo que en futuras ediciones habría que incidir más en la explicación de sus características, su manejo y uso. La Fig. 12 muestra los resultados obtenidos para el Bloque 1 de la encuesta.

\section{Preguntas del Bloque 1}
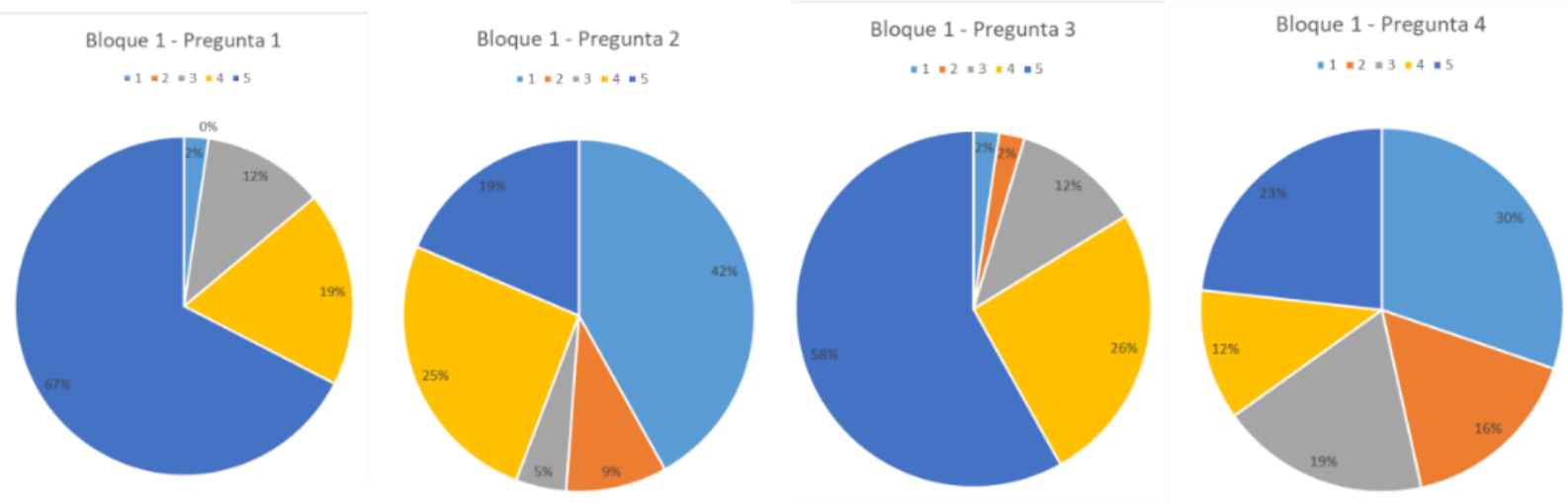

Fig. 12. Resultados del Bloque 1. [Elaboración propia].

Por otro lado, los resultados obtenidos para el Bloque 2 (Material y entorno) muestran una gran satisfacción con el jurado y personal de apoyo, ya que se han obtenido valoraciones de satisfacción máxima superiores al $70 \%$ en todos los apartados. También se encuentran muy satisfechos con las instalaciones y herramientas utilizadas durante la actividad. Esto nos permite evaluar la ergonomía del espacio donde se ha llevado a cabo la prueba y la idoneidad de los equipos que se han dispuesto para la realización de las pruebas. La Fig. 13 muestra los resultados obtenidos para el Bloque 2 de la encuesta.

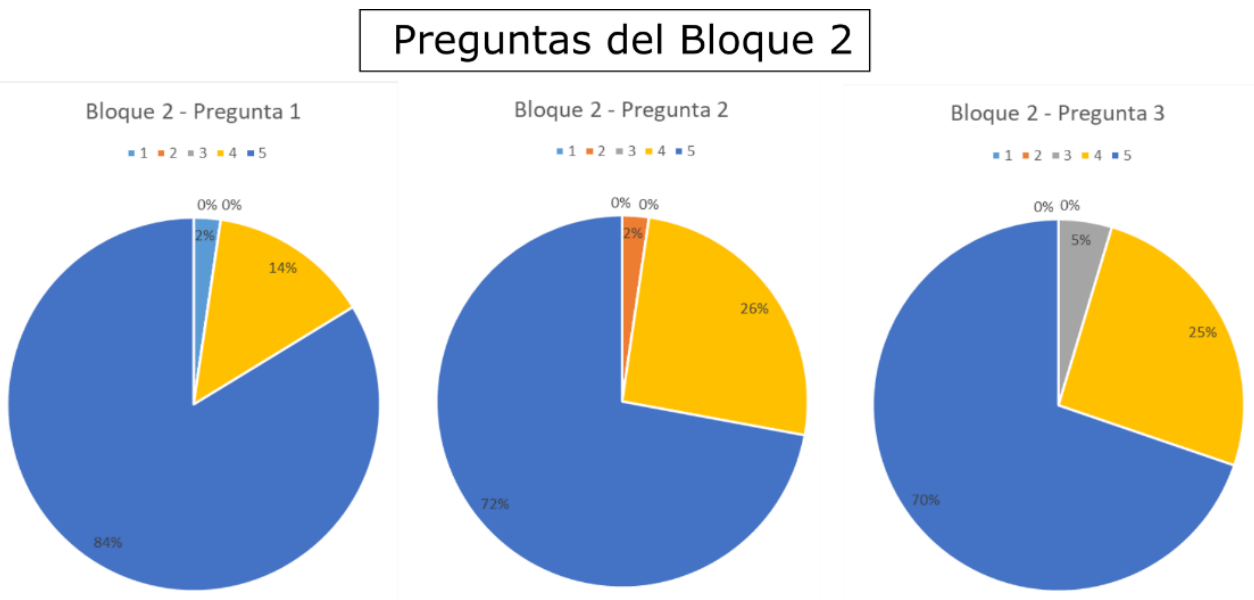

Fig. 13. Resultados del Bloque 2. [Elaboración propia].

Por último, un $67 \%$ de los alumnos ha considerado divertida la actividad práctica desarrollada, mientras que un $82 \%$ de ellos la recomendaría. Esto nos indica que la actividad escogida para acercar la Ingeniería Civil a alumnos de secundaria es atractiva para ellos, por lo que podría ser interesante su repetición en ediciones futuras. La Fig. 14 muestra los resultados obtenidos del Bloque 3 (otros aspectos). 


\section{Preguntas del Bloque 3}

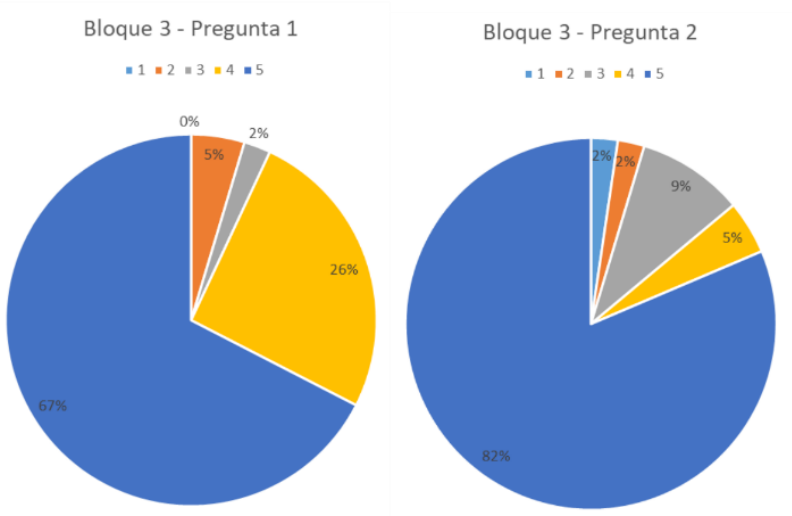

Fig. 14. Resultados del Bloque 3. [Elaboración propia].

\section{Conclusiones}

El presente artículo plantea la construcción de una presa de materiales sueltos a escala y una maqueta de puente con palillos de madera por parte de estudiantes de secundaria. El objetivo que se persigue es acercar la Ingeniería Civil a los alumnos que se encuentran a las puertas de acceso a la universidad, mostrándoles una de las aplicaciones que podrían desarrollar en un futuro como ingenieros civiles. Gracias a la implementación de esta gymkana a modo de concurso, se animaba a los alumnos a la proactividad durante la prueba y a que interactuasen entre ellos.

Para la fabricación del talud, se ha dispuesto de una serie de materiales granulares (arcilla y gravas de diferentes granulometrías) que debían utilizar en capas sucesivas para conformar el talud en el interior de un cajón transparente. Posteriormente, y transcurrido un tiempo máximo de cinco minutos, el jurado de la prueba vertía agua en uno de los paramentos de la presa y se cronometraba el tiempo de filtración de agua. El equipo que más tiempo tardase en filtrar el agua a través del talud resultaba ganador de la prueba.

Las diferentes maquetas de puente eran expuestas y presentadas ante el público asistente por parte de sus propios autores, hecho que un grupo de docentes designados tuvo en cuenta para otorgar el premio del jurado. Posteriormente, cada uno de los modelos fue sometido a una prueba de carga mediante la colocación de adoquines sobre el tablero del puente. El objetivo era alcanzar el peso máximo que podía resistir la estructura sin llegar al colapso.

Tras la realización de la actividad se entregaron una serie de formularios a los alumnos en donde se quería evaluar el grado de satisfacción de la prueba que habían desarrollado. Los resultados obtenidos muestran que la actividad práctica fue de su agrado, que consiguieron entender el alcance de la tarea y que recomendarían sin duda la realización de la prueba en ediciones sucesivas. Además, tras analizar los resultados obtenidos según edades de los participantes se ha podido comprobar que los estudiantes más jóvenes han valorado la actividad como mucho más divertida que los participantes de más edad, pero éstos últimos han valorado mucho más positivamente las explicaciones y el objetivo final de la prueba. De este modo, se podría constatar que los alumnos más cercanos al acceso a la universidad valoran más los conocimientos y comprensión de los problemas que lo ameno de la actividad, mostrando una actitud mucho más madura. Estos datos permitirán mejorar ciertos aspectos a futuro y, también, corroborar que la actividad cumple con el objetivo marcado. 


\section{Agradecimientos}

Las pruebas de construcción de una presa-dique y la prueba de carga sobre maqueta de puente se llevaron a cabo durante la II Olimpiada Universitaria en Ingeniería, realizada en la Escuela Politécnica de Mieres (EPM) por institutos de educación secundaria del Principado de Asturias en el marco de las jornadas de acercamiento y puertas abiertas de la EPM para estudiantes pre-universitarios.

\section{Referencias}

\section{Artículo de una revista o periódico}

ALFARO, FJ., PARDO, JJ., MOLINA, JP., GARCÍA, PJ. (2011). "La promoción de los estudios de informática mediante la olimpiada de informática". XVII Jornadas de Enseñanza Universitaria de la Informática.

CAMPOS-ARANDA, DF. (2013). "Modelado empírico simple del rompimiento de presas pequeñas de tierra (hidrograma de salidas)". Ingeniería y Tecnología, volumen XIV (número 3).

LACUESTA, R., PALACIOS, G., FERNÁNDEZ, L. (2009). “Active learning through problem based learning methodology in engineering education". Proceedings: 39th ASEE/IEEE Frontiers in Eduaction Conference, San Antonio, Texas.

LEÓN, MJ., CRISOL, E. (2011). “Diseño de cuestionarios (Oppumaugr y Opeaumaugr): la opinión y la percepción del profesorado y de los estudiantes sobre el uso de las metodologías activas en la universidad”. Revista de Curriculum y Formación del Profesorado,13(1), 305-319.

PRINCE, M. (2004). "Does active learning work? A review of the research". Journal of Engineering Education, 93(3), 223-231.

SANTOS-AMADO, JD., MALAGÓN-VILLAFRADES, PY., CÓRDOBA-YUTA, EM. (2011). "Caracterización de arcillas y preparación de pastas cerámicas para la fabricación de tejas y ladrillos en la región de Barichara, Santander". DYNA, Vol 78, Num 167.

Página web

ASOCIACIÓN CHILENA DE ESTUDIANTES DE INGENIERÍA (ACHEII). 2016. Ver: http://www.userena.cl/actualidad/1912-estudiantes-de-la-uls-organizan-olimpiadas-deingenier\%C3\%ADa-civil-industrial.html [Acceso 21 de Julio de 2020].

CITOPIC. Definición. Ver: https://www.citop.es/colegio/colegio.php?id=48 [Acceso 21 de Julio de 2020].

ESTUDIOS SASSANI. INGENIERÍA ESTRUCTURAL. (2018). Ver: https://estudiossassani.wordpress.com/2018/07/30/colapsa-pasarela-atirantada-en-bogota-durante-laprueba-de-carga/ [Acceso 21 de Julio de 2020].

GRUPO DE INVESTIGACIÓN DE MATEMÁTICAS APLICADAS A LA INGENIERÍA CIVIL (MAIC) DEL DEPARTAMENTO DE MATEMÁTICAS E INFORMÁTICA APLICADA A LA INGENIERÍA CIVIL DE LA ESCUELA POLITÉCNICA DE MADRID (UPM). 2010. Ver: https://www2.caminos.upm.es/Departamentos/matematicas/Fdistancia/PIE/innovacion.htm [Acceso 21 de Julio de 2020].

UNIVERSIDAD MICHOACANA DE SAN NICOLÁS DE HIDALGO (UMSNH). 2018. Ver: https://www.cic.umich.mx/noticias/158-arrasa-umsnh-en-olimpiada-nacional-de-ingenieria-civil.html [Acceso 21 de Julio de 2020]. 
UNIVERSIDAD POLITÉCNICA DE CARTAGENA $\quad$ (UPCT). 2019. Ver: https://repositorio.upct.es/handle/10317/7767 [Acceso 21 de Julio de 2020].

UNIVERSIDAD POLITÉCNICA DE CARTAGENA (UPCT). 2020. Ver: https://caminosyminas.upct.es/olimpiadas-caminos-2020 [Acceso 21 de Julio de 2020].

YEPES, V. (2017). EL BLOG DE VÍCTOR YEPES. CONCEPTO DE PUENTE VIGA Y ALGO DE HISTORIA. Ver: https://victoryepes.blogs.upv.es/ [Acceso 21 de Julio de 2020].

\section{Legislación y normas}

España. Diccionario RAE. Definición de Ingeniería Civil.

España. Instrucción sobre las acciones a considerar en el proyecto de puentes de ferrocarril IAPF-07. 2007. Ministerio de Fomento.

España. Recomendaciones para la realización de pruebas de carga de recepción en puentes de carretera. 1999. Ministerio de Fomento. 